\title{
Factors associated with anti-tumor necrosis factor effectiveness to prevent postoperative recurrence in Crohn's disease
}

\author{
Anthony Buisson ${ }^{1,2,3}$, Lisa Cannon ${ }^{4}$, Konstantin Umanskiy $^{4}$, Roger D. Hurst ${ }^{4}$, Neil H. Hyman ${ }^{4}$, Atsushi Sakuraba $^{1}$, \\ Joel Pekow ${ }^{1}$, Sushila Dalal ${ }^{1}$, Russell D. Cohen ${ }^{1}$, Bruno Pereira ${ }^{5}$, David T. Rubin ${ }^{1}$ \\ ${ }^{1}$ Inflammatory Bowel Disease Center, University of Chicago Medicine, Chicago, IL, USA; ${ }^{2}$ Inflammatory Bowel Unit, Inserm, 3iHP, CHU \\ Clermont-Ferrand, Clermont Auvergne University, Clermont-Ferrand; ${ }^{3}$ USC INRae 2018, M2iSH, Inserm U1071, 3iHP, Clermont Auvergne \\ University, Clermont-Ferrand, France; ${ }^{4}$ Department of Surgery, University of Chicago Medicine, Chicago, IL, USA; ${ }^{5}$ Biostatistics Unit, DRCI, \\ CHU Clermont-Ferrand, Clermont Auvergne University, Clermont-Ferrand, France
}

Background/Aims: We assessed the effectiveness of anti-TNF agents and its associated factors to prevent endoscopic and clinical postoperative recurrence (POR) in Crohn's disease (CD). Methods: From a prospectively-maintained database, we retrieved 316 CD patients who underwent intestinal resection (2011-2017). Endoscopic (Rutgeerts index $\geq i 2$ at 6 months) and clinical (recurrence of symptoms leading to hospitalization or therapeutic escalation) POR were assessed. Results: In 117 anti-TNF-naïve patients, anti-TNF therapy was more effective than immunosuppressive agents (odds ratio [OR], 8.8; 95\% confidence interval [CI], 1.8-43.9; $P=0.008)$ and no medication/5-aminosalicylates (OR, 5.2; 95\% CI, 1.0-27.9; $P=0.05)$ to prevent endoscopic POR. In 199 patients exposed to anti-TNF prior to the surgery, combination with anti-TNF and immunosuppressive agents was more effective than anti-TNF monotherapy (OR, 2.32; 95\% CI, 1.02-5.31; $P=0.046)$ to prevent endoscopic POR. Primary failure to anti-TNF agent prior to surgery was predictive of anti-TNF failure to prevent endoscopic POR (OR, 2.41; 95\% CI, 1.10-5.32; $P=0.03)$. When endoscopic POR despite anti-TNF prophylactic medication $(\mathrm{n}=55)$, optimizing anti-TNF and adding an immunosuppressive drug was the most effective option to prevent clinical POR (hazard ratio, 7.38; 95\% CI, 1.54-35.30; $P=0.012$ ). Anti-TNF therapy was the best option to prevent clinical POR (hazard ratio, $3.10 ; 95 \% \mathrm{CI}, 1.09-8.83 ; P=0.034$ ) in patients with endoscopic POR who did not receive any biologic to prevent endoscopic POR $(n=55)$. Conclusions: Anti-TNF was the most effective medication to prevent endoscopic and clinical POR. Combination with anti-TNF and immunosuppressive agents should be considered in patients previously exposed to anti-TNF. (Intest Res 2022;20:303-312)

Key Words: Inflammatory bowel disease; Surgery; Ileocolonic resection; Combination therapy

\section{INTRODUCTION}

Crohn's disease (CD) is a disabling and progressive disorder involving the digestive tract that can lead to bowel damage as

Received February 5, 2021. Revised May 19, 2021. Accepted May 30, 2021. Correspondence to David T. Rubin, Inflammatory Bowel Disease Center, University of Chicago Medicine, 5841 S. Maryland Ave. MC 4076, Chicago, IL 60634, USA. Tel: +1-773-702-2950, Fax: +1-773-702-2182, E-mail:

drubin@medicine.bsd.uchicago.edu well as altered quality of life and high disability for patients. ${ }^{1,2}$ Despite the larger use of biologics, bowel resection is required in almost one half of the patients. ${ }^{3}$ After surgery, the reappearance of endoscopic lesions without symptoms, so-called endoscopic postoperative recurrence (POR), is very common, as $75 \%$ of the patients from referral centers have been reported to experience POR within the first year after surgery. ${ }^{4}$ Endoscopic POR assessed by the Rutgeerts index (RI) is highly predictive of clinical POR, i.e., reoccurrence of CD-related symp- 
toms. ${ }^{5,6}$ The management of the postoperative period has evolved in recent years. It is currently recommended to perform a systematic colonoscopy at 6 months to assess the RI leading to therapeutic escalation in case of endoscopic POR defined as $\mathrm{RI} \geq \mathrm{i} 2{ }^{7}$ In addition, most patients receive a prophylactic medication within the first month after surgery to prevent endoscopic POR. We recently reported that these modern strategies may have contributed to a decreased rate of POR. ${ }^{6}$ However, the decision of starting a prophylactic medication should consider the risk of POR but also the risk of overtreatment for some patients ( $10 \%$ to $15 \%$ in the historical cohort described by Rutgeerts et al. ${ }^{5}$ ) who will not recur despite the absence of preventive treatment. Although several options are available, anti-tumor necrosis factor (TNF) agents are considered the most effective medications to prevent and to treat endoscopic POR in $\mathrm{CD}^{8.14}$ However, up to $30 \%$ of the patients experienced anti-TNF failure in this situation highlighting the need to identify predictors of therapeutic efficacy. ${ }^{8-14}$

In this study, we assessed the effectiveness of anti-TNF agents compared to other medications and identified the factors associated with the effectiveness of anti-TNF agents to prevent endoscopic or clinical POR in patients with CD.

\section{METHODS}

\section{Ethical Consideration}

This study was performed in accordance with the Declaration of Helsinki, Good Clinical Practice and applicable regulatory requirements. The study was approved by the Institutional Review Board of the University of Chicago (IRB No. 16-0061). The informed consent was waived.

\section{Patients and Study Design}

From a prospectively-maintained database (Genesys, IRB protocol 15573A), we identified a cohort of consecutive patients with $\mathrm{CD}$ who underwent ileocolonic resection and anastomosis between January 2011 and June 2017 at the University of Chicago Medicine Inflammatory Bowel Disease Center. ${ }^{6}$ All the adult patients ( $\geq 18$ years) with CD were consecutively screened and were eligible to participate if: (1) they underwent ileocolonic resection (patients with isolated ileal or colonic resection as well as stricturoplasty was not included); (2) all macroscopic lesions were removed; (3) the anastomosis was reachable by ileocolonoscopy; (4) a systematic colonoscopy was performed at 6 months following surgery; or (5) the follow-up was longer than 6 months after bowel resection. ${ }^{6}$

\section{Management of Postoperative Period}

The postoperative management of patients with $\mathrm{CD}$ is standardized in the University of Chicago Medicine Inflammatory Bowel Disease Center. A prophylactic medication is started in most of the patients according to the risk factors and based on physician's assessment. A colonoscopy is proposed for all the patients at 6 months. The endoscopic evaluation was performed by experienced inflammatory bowel disease endoscopists and graded according to the RI. ${ }^{5}$ In the case of a RI $\geq i 2$, a therapeutic intensification was performed.

\section{Medications, Investigated Factors and Study Endpoints}

Anti-TNF agents were used at the usual dose and regimen, even for patients who have been treated with anti-TNF therapies before surgery (re-induction): adalimumab (160 mg at week $0,80 \mathrm{mg}$ at week 2 and $40 \mathrm{mg}$ every other week), certolizumab pegol ( $400 \mathrm{mg}$ at week 0 , week 2 , week 4 and every 4 weeks) and golimumab (200 mg at week $0,100 \mathrm{mg}$ at week 2 and $100 \mathrm{mg}$ every 4 weeks $)$ and infliximab $(5 \mathrm{mg} / \mathrm{kg}$ at week 0 , week 2, week 6 and every 8 weeks). Anti-TNF agents were started within the first month after surgery. Therapeutic drug monitoring was not systematically performed at that time in our center. The doses of azathioprine and 6-mercaptopurine were $2-2.5 \mathrm{mg} / \mathrm{kg}$ and $1-1.5 \mathrm{mg} / \mathrm{kg}$ once daily, respectively. Methotrexate was used as subcutaneous monotherapy (25 mg weekly for 3 months followed by $15 \mathrm{mg}$ weekly) or per os (10$15 \mathrm{mg}$ weekly) in combination therapy with anti-TNF. Metronidazole was given at low dose (250 mg 3 times daily) until endoscopic evaluation.

Anti-TNF primary failure was defined as absence of clinical response despite a complete induction regimen with appropriate dose. Surgery for refractoriness to medical therapy is defined as bowel resection performed due to failure to all the available medications despite the absence of stricture or fistula.

Endoscopic POR was defined as RI $\geq i 2$. According to previous publications, ${ }^{15,16}$ clinical POR was defined as recurrence of symptoms (Harvey-Bradshaw index $>4$ ) leading to hospitalization or therapeutic intensification after exclusion of other causes of recurrent symptoms such as bile-salt diarrhea, bacterial overgrowth and adhesion-related obstruction.

\section{Data management and Statistical Analysis}

Study data were collected and managed using REDCap (Research Electronic Data Capture) tools, which is a secure, webbased application designed to support data capture for research studies, providing (1) an intuitive interface for validated data 
entry; (2) audit trails for tracking data manipulation and export procedures; (3) automated export procedures for seamless data downloads to common statistical packages; and (4) procedures for importing data from external sources.

Statistical analysis was performed using Stata 13 software (StataCorp LP, College Station, TX, USA). The tests were twosided, with a type I error set at $5 \%$. Subject's characteristics were presented as mean \pm standard deviation or median (interquartile range) for continuous data (assumption of normality assessed using the Shapiro-Wilk test) and as the number of patients and associated percentages for categorical parameters. Comparisons between the independent groups were performed using the chi-square or Fisher exact tests for categorical variables, and using Student $t$-test or Mann-Whitney test for quantitative parameters (normality, assumption of homoscedasticity studied using Fisher-Snedecor test).

For the analyses of endoscopic risk factors of POR, we performed univariate and multivariable analyses (logistic regression according to univariate results and clinical relevance). The results were expressed as odds ratio (OR) and 95\% confidence intervals (CIs).

For the analysis of clinical POR (censored data), estimates were constructed using the Kaplan-Meier method. The logrank test was used in a univariate analysis to test the prediction of each parameter for the occurrence of an event. Cox proportional hazards regression was used to investigate risk factors in a multivariable situation by backward and forward stepwise analysis of the factors considered significant in univariate analysis (entered into the model if $P<0.10$ ) and according to clini- cal relevance. Results were expressed as hazard ratios (HRs) and $95 \%$ CIs. The proportional-hazard hypothesis was studied by Schoenfeld test.

\section{RESULTS}

\section{Baseline Characteristics of the Patients}

The characteristics of the 316 patients were detailed in Table 1 distinguishing anti-TNF naïve and previously anti-TNF-exposed patients at the time of surgery (Fig. 1).

\section{Prevention of Endoscopic POR 1) Anti-TNF-Nä̈ve Patients (117 Patients)}

Overall, 23 patients received anti-TNF agents (infliximab [n=10] and adalimumab $[\mathrm{n}=13]$ ) including 5 patients on infliximab monotherapy, 6 patients on adalimumab monotherapy, 5 patients with infliximab+azathioprine, 3 patients with adalimumab+ azathioprine, 1 patient with adalimumab+6-mercaptopurine, and 3 patients with adalimumab+methotrexate. In univariate analysis, the rate of endoscopic POR was $8.7 \%(2 / 23)$ in patients receiving anti-TNF to prevent endoscopic POR. Among them, $9.0 \%(1 / 11)$ treated with monotherapy experienced endoscopic POR while 8.3\% (1/12) receiving concomitant immunosuppressants had endoscopic POR. The rate of endoscopic POR was $40.4 \%$ (23/57) in patients on immunosuppressive agents (azathioprine [n=40], 6-mercaptopurine [ $\mathrm{n}=14]$, and methotrexate $[\mathrm{n}=3])$ and $32.4 \%(12 / 37)$ in patients with no medication $(\mathrm{n}=30)$ or treated with 5-aminosalicylates (5-ASA) $(n=4)$ or metronidazole monotherapy $(n=3)$.

Table 1. Characteristics of the 316 Patients with Crohn's Disease at the Time of Surgery

\begin{tabular}{|c|c|c|c|}
\hline Characteristics & $\begin{array}{l}\text { Overall population } \\
\qquad(n=316)\end{array}$ & $\begin{array}{l}\text { Anti-TNF naïve patients } \\
\qquad(n=117)\end{array}$ & $\begin{array}{l}\text { Anti-TNF-exposed patients } \\
\qquad(\mathrm{n}=199)\end{array}$ \\
\hline Age at the time of surgery (yr) & $38.8 \pm 14.2$ & $40.8 \pm 15.4$ & $37.7 \pm 13.4$ \\
\hline Female sex & $160(50.6)$ & $66(56.4)$ & $94(47.2)$ \\
\hline Smokers & $37(11.7)$ & $16(13.7)$ & $21(10.5)$ \\
\hline \multicolumn{4}{|l|}{ Location } \\
\hline L1 & $113(35.8)$ & $51(43.6)$ & $62(31.2)$ \\
\hline L2 & $18(5.7)$ & $5(4.3)$ & $13(6.5)$ \\
\hline L3 & $185(58.5)$ & $61(52.1)$ & $124(62.3)$ \\
\hline
\end{tabular}


Table 1. Continued

\begin{tabular}{|c|c|c|c|}
\hline Characteristics & $\begin{array}{l}\text { Overall population } \\
\quad(\mathrm{n}=316)\end{array}$ & $\begin{array}{l}\text { Anti-TNF naïve patients } \\
\qquad(\mathrm{n}=117)\end{array}$ & $\begin{array}{l}\text { Anti-TNF-exposed patients } \\
\qquad(\mathrm{n}=199)\end{array}$ \\
\hline \multicolumn{4}{|l|}{ Behavior } \\
\hline B1 & $20(6.3)$ & $7(6.0)$ & $13(6.7)$ \\
\hline B2 & $152(48.1)$ & $53(45.3)$ & $99(49.7)$ \\
\hline B3 & $144(45.6)$ & $57(48.7)$ & $87(43.7)$ \\
\hline Prior intestinal resection & $119(37.7)$ & $36(31.0)$ & $83(41.7)$ \\
\hline \multicolumn{4}{|l|}{ Medications before surgery } \\
\hline Thiopurines & $177(55.7)$ & $66(57.4)$ & $151(76.3)$ \\
\hline Methotrexate & $50(15.8)$ & $6(5.1)$ & $44(22.1)$ \\
\hline Anti-TNF-naïve & $117(37.0)$ & $117(100)$ & 0 \\
\hline 1 Biologic & $107(33.9)$ & 0 & $107(53.8)$ \\
\hline 2 Biologics & 54 (17.1) & 0 & $54(27.1)$ \\
\hline 3 Biologics & $25(7.9)$ & 0 & $25(12.6)$ \\
\hline 4 Biologics & $9(2.8)$ & 0 & $9(4.5)$ \\
\hline 5 Biologics & $4(1.2)$ & 0 & $4(2.0)$ \\
\hline Infliximab & $137(43.3)$ & 0 & $137(68.9)$ \\
\hline Adalimumab & $135(42.7)$ & 0 & $135(67.8)$ \\
\hline Certolizumab pegol & 48 (15.2) & 0 & 48 (24.1) \\
\hline Vedolizumab & $16(5.0)$ & 0 & $16(8.0)$ \\
\hline Ustekinumab & $4(1.2)$ & 0 & $4(2.0)$ \\
\hline Primary failure to any anti-TNF agents & $96(30.4)$ & 0 & $96(48.2)$ \\
\hline Intestinal resection length $(\mathrm{cm})$ & $22.0(11.5-35.1)$ & $29.0(19.0-43.5)$ & $36.5(22.5-54.8)$ \\
\hline Intestinal resection length $>30 \mathrm{~cm}$ & $170(53.8)$ & $54(46.2)$ & $116(58.3)$ \\
\hline \multicolumn{4}{|l|}{ Indication for surgery } \\
\hline Failure to medical therapy & $7(2.2)$ & $2(1.7)$ & $5(2.5)$ \\
\hline Stricture & $184(52.8)$ & $62(53.0)$ & $122(61.3)$ \\
\hline Fistula/abscess & $119(37.6)$ & $51(43.6)$ & 68 (34.2) \\
\hline Cancer/dysplasia & $6(1.9)$ & $2(1.7)$ & $4(2.0)$ \\
\hline \multicolumn{4}{|l|}{ Medications after surgery } \\
\hline No treatment & $41(13.0)$ & $27(23.1)$ & $14(7.0)$ \\
\hline 5-ASA & $7(2.2)$ & $5(4.3)$ & $2(1.0)$ \\
\hline Thiopurines & $131(41.0)$ & $48(41.0)$ & $47(23.6)$ \\
\hline Methotrexate & 41 (15.8) & $6(5.1)$ & $35(17.6)$ \\
\hline Metronidazole & $38(12.0)$ & $11(9.4)$ & $27(13.6)$ \\
\hline Anti-TNF agents & $148(46.8)$ & $23(19.7)$ & $125(62.8)$ \\
\hline Infliximab & $45(14.2)$ & $10(8.5)$ & $35(17.6)$ \\
\hline Adalimumab & $91(28.8)$ & $13(11.0)$ & $78(39.2)$ \\
\hline Certolizumab pegol & $10(3.2)$ & 0 & $10(5.0)$ \\
\hline Golimumab & $1(0.3)$ & 0 & $1(0.5)$ \\
\hline Anti-TNF combotherapy & $76(24.1)$ & $12(10.2)$ & $64(32.2)$ \\
\hline Vedolizumab & $18(5.7)$ & $3(2.5)$ & $15(7.5)$ \\
\hline Ustekinumab & $4(1.2)$ & 0 & $4(2.0)$ \\
\hline
\end{tabular}

Values are presented as mean $\pm S D$, median (IQR), or number $(\%)$.

TNF, tumor necrosis factor; 5-ASA, 5-aminosalicylates; SD, standard deviation; IQR, interquartile range. 


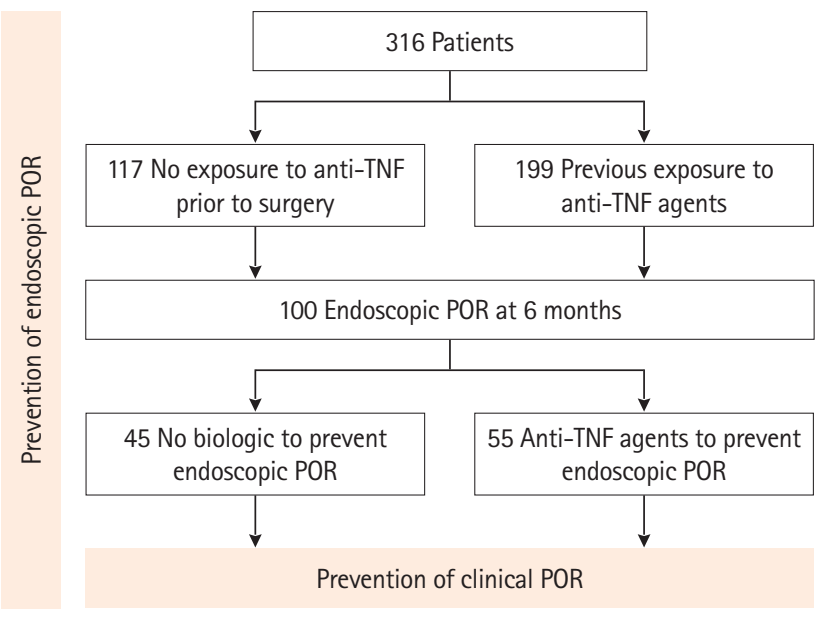

Fig. 1. Flowchart showing the sample size of each subgroup of investigated patients. TNF, tumor necrosis factor; POR, postoperative recurrence.

In multivariable analysis (Supplementary Table 1) adjusted on usual risk factors of endoscopic POR, ${ }^{6}$ i.e. active cigarette smoker, perianal lesions, fistulizing phenotype (B3 according to Montreal classification), resection length $>30 \mathrm{~cm}$, prior history of intestinal resection, surgery for refractoriness to medical therapy and use of metronidazole as prophylactic medication, anti-TNF therapy was more effective than immunosuppressive agents (OR, 8.8; 95\% CI, 1.8-43.9; $P=0.008$ ) and no medication/5-ASA (OR, 5.2; 95\% CI, 1.0-27.9; $P=0.05$ ) (Fig. 2).

\section{2) Patients Exposed to Anti-TNF Prior to the Surgery (199 Patients)}

In the group of patients exposed to anti-TNF agents before surgery, 64 patients received combination therapy with antiTNF and immunosuppressive agents (infliximab+thiopurines = 13 patients, infliximab + methotrexate $=6$ patients, adalimumab + thiopurines $=25$ patients, and adalimumab + methotrexate $=$ 20 patients) to prevent endoscopic POR. Among them, 25.0\% (16/64) had endoscopic POR at 6 months. On the other hand, 61 patients were treated with anti-TNF monotherapy including 16 with infliximab, 33 with adalimumab, 11 with certolizumab pegol and 1 with golimumab. Among them, 44.3\% (27/61) experienced endoscopic POR at 6 months. In our cohort, 50.0\% $(11 / 22)$ of the patients receiving anti-integrin therapies experienced endoscopic POR at 6 months including 46.6\% (7/15) and $57.1 \%(4 / 7)$ for vedolizumab and natalizumab, respectively. None $(0 / 4)$ of the patients treated with ustekinumab had endoscopic POR at 6 months. The rate of endoscopic POR was $45.8 \%(22 / 48)$ in patients with no medication or conventional

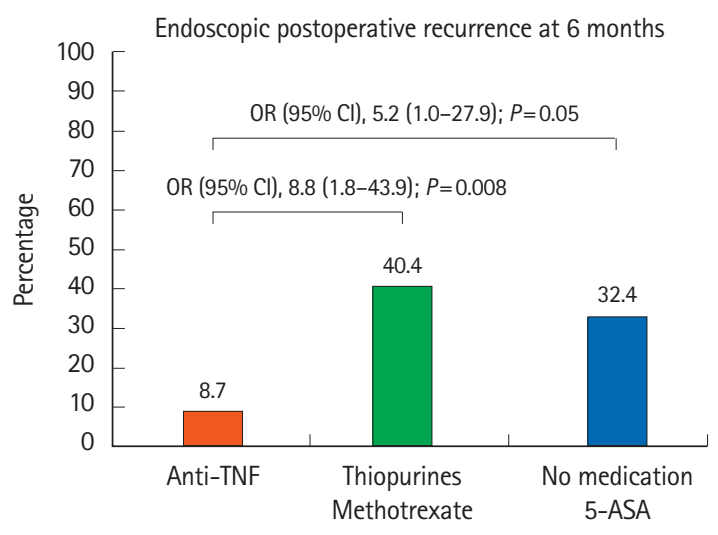

Fig. 2. Comparison of therapeutic effectiveness to prevent endoscopic postoperative recurrence in 117 anti-TNF-naïve patients with Crohn's disease. $\mathrm{OR}$ and $P$-values are given in multivariable analyses with $95 \% \mathrm{Cl}$. OR, odds ratio; $\mathrm{Cl}$, confidence interval; TNF, tumor necrosis factor; 5-ASA, 5-aminosalicylates.

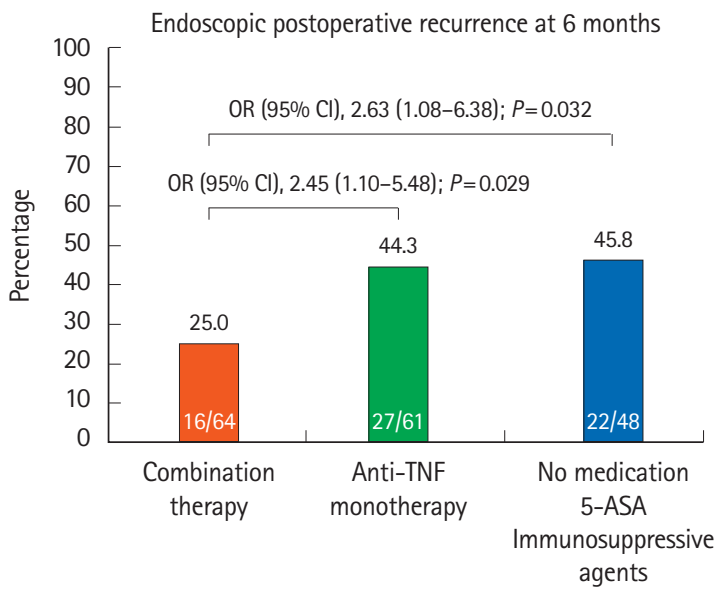

Fig. 3. Comparison of therapeutic effectiveness to prevent endoscopic postoperative recurrence in 199 patients with Crohn's disease exposed to anti-TNF before surgery. OR and $P$-values are given in multivariable analyses with $95 \% \mathrm{Cl}$. OR, odds ratio; $\mathrm{Cl}_{\text {, }}$ confidence interval; TNF, tumor necrosis factor; 5-ASA, 5-aminosalicylates.

therapy (thiopurines, methotrexate, and 5-ASA).

In multivariable analysis ( $\mathrm{n}=199)$ (Supplementary Table 2) adjusted on gender, active cigarette smokers, perianal lesions, fistulizing phenotype (B3 according to Montreal classification), resection length $>30 \mathrm{~cm}$, prior bowel resection, surgery for refractoriness to medical therapy, number of biologics prior to surgery, combination therapy with anti-TNF and immunosuppressive agents was more effective than anti-TNF monotherapy (OR, $2.45 ; 95 \% \mathrm{CI}, 1.10-5.48 ; P=0.029)$ or conventional medications (no medication, 5-ASA or immunosuppressive monotherapy) (OR, 2.63 ; $95 \% \mathrm{CI}, 1.08-6.38$; $P=0.032$ ) to pre- 
vent endoscopic POR at 6 months (Fig. 3) but failed to show any significant difference compared to anti-integrin therapies (OR, 1.87; 95\% CI, 0.53-6.66; $P=0.333$ ) or ustekinumab (OR, $0.22 ; 95 \%$ CI, 0.01-6.68; $P=0.383$ ).

In multivariable analysis $(n=125)$, the factors associated with anti-TNF failure were anti-TNF monotherapy (OR, 2.34; 95\% CI, 1.01-5.43; $P=0.046$ ), primary failure to at least 1 antiTNF agent prior to surgery (OR, 2.41; 95\% CI, 1.10-5.32; $P=$ 0.030 ), male gender (OR, 2.86; 95\% CI, 1.32-6.20; $P=0.008$ ) and surgery for refractoriness to medical therapy (OR, 31.6; 95\% CI, 1.03-96.81; $P=0.048$ ) (Table 2). In contrast, resuming the same anti-TNF agent than before the surgery $(107 / 125$ : OR, $0.75 ; 95 \%$ CI, $0.24-2.43 ; P=0.638$ ) even in the case of primary nonresponse with this specific anti-TNF (33/107: OR, 0.50; 95\% CI, 0.17-1.48; $P=0.209$ ) and more than 2 anti-TNF agents prior to surgery (42/125: OR, 2.40; 95\% CI, 0.79-7.30; $P=0.120$ ) were not significantly associated with higher rate of endoscopic POR (Table 2).

Table 2. Anti-TNF Effectiveness to Prevent Endoscopic POR: Subgroups of Patients with Anti-TNF Exposure Prior to Surgery

\begin{tabular}{|c|c|c|}
\hline Factor & OR (95\% Cl) & $P$-value \\
\hline Active smoker & $0.383(0.104-1.415)$ & 0.150 \\
\hline Perianal lesions & $1.972(0.844-4.609)$ & 0.117 \\
\hline Prior bowel resection & $0.858(0.411-1.795)$ & 0.685 \\
\hline $\begin{array}{l}\text { More than } 2 \text { anti-TNF before } \\
\text { surgery }\end{array}$ & $2.408(0.794-7.303)$ & 0.120 \\
\hline $\begin{array}{l}\text { Primary nonresponder to } \\
1 \text { anti-TNF before surgery }\end{array}$ & $2.410(1.091-5.324)$ & 0.030 \\
\hline $\begin{array}{l}\text { Resuming the same anti-TNF than } \\
\text { before surgery }\end{array}$ & $0.755(0.235-2.430)$ & 0.638 \\
\hline $\begin{array}{l}\text { Resuming the anti-TNF for which } \\
\text { he was primary nonresponder }\end{array}$ & $0.500(0.170-1.475)$ & 0.209 \\
\hline Resection length $>30 \mathrm{~cm}$ & $1.669(0.802-3.474)$ & 0.171 \\
\hline $\begin{array}{l}\text { Surgery for refractoriness to } \\
\text { medical therapy }\end{array}$ & $31.597(1.031-96.816)$ & 0.048 \\
\hline $\begin{array}{l}\text { Metronidazole to prevent } \\
\text { endoscopic POR }\end{array}$ & $0.930(0.307-2.820)$ & 0.898 \\
\hline Fistulizing phenotyp (B3) & $0.711(0.332-1.522)$ & 0.379 \\
\hline Combination therapy & Reference & \\
\hline Anti-TNF monotherapy & $2.347(1.014-5.429)$ & 0.046 \\
\hline Female sex & $2.858(1.318-6.198)$ & 0.008 \\
\hline
\end{tabular}

TNF, tumor necrosis factor; $\mathrm{POR}$, postoperative recurrence; $\mathrm{OR}$, odds ratio; $\mathrm{Cl}$, confidence interval.

\section{Prevention of Clinical POR \\ 1) Patients Who Had Endoscopic POR Despite Anti-TNF \\ Agents as Prophylactic Medications (45 Patients)}

Among the 45 patients with early use of anti-TNF agents to prevent endoscopic POR and who had endoscopic POR at 6 months, infliximab was started in 9 patients (3 with concomitant thiopurines and 1 with methotrexate), adalimumab was started in 29 patients ( 7 with concomitant thiopurines and 6 with methotrexate), certolizumab pegol in 6 patients ( 1 with concomitant thiopurines and 1 with methotrexate) and 1 was treated with golimumab and methotrexate. During a mean follow-up of $18.7 \pm 12.4$ months, clinical POR occurred in $68.9 \%$ (31/45) of these patients. A multivariable analysis (Supplementary Table 3) adjusted on active cigarette smokers, gender, prior history of bowel resection, exposure or primary failure to antiTNF before surgery and fistulizing phenotype (B3 according to Montreal classification) showed that therapeutic escalation combining increased dose/shortened interval of the same anti-TNF and addition of immunosuppressive medication was a more effective option to prevent clinical POR than other strategies including increased dose/shortened interval alone, switch to another anti-TNF or swap to vedolizumab (HR, $7.38 ; 95 \% \mathrm{CI}$, 1.54-35.30; $P=0.012$ ) (Fig. 4). In the same statistical model, severe endoscopic POR (RI, i3 or i4) was also associated with higher risk of clinical POR (HR, 3.12; 95\% CI, 1.17-8.32; $P=0.023)$.

\section{2) Patients Who Did Not Receive Any Biologic to Prevent Endoscopic POR (55 Patients)}

Among the 55 patients who were not treated with biologics to

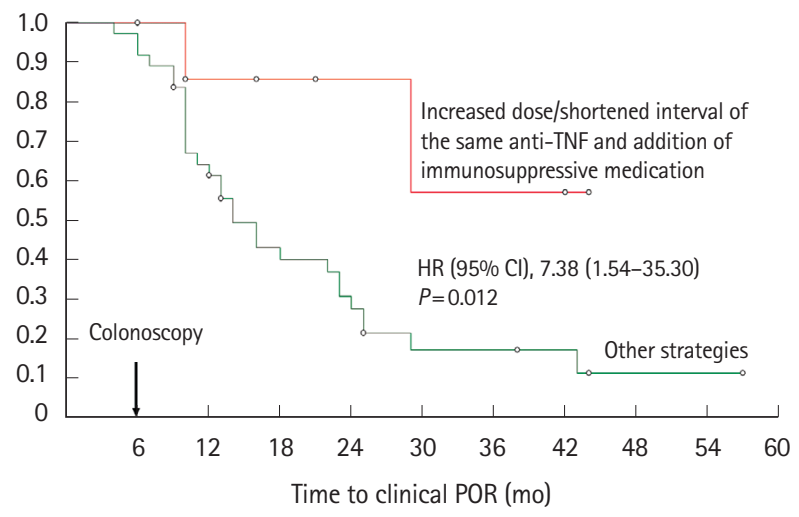

Fig. 4. Kaplan-Meier curve comparing the risk of clinical POR according to therapeutic strategies in patients who had endoscopic POR at 6 months despite anti-TNF therapy. HR and $P$-values are given in multivariable analyses with $95 \% \mathrm{Cl}$. $\mathrm{HR}$, hazard ratio; $\mathrm{Cl}$, confidence interval; TNF, tumor necrosis factor; POR, postoperative recurrence. 


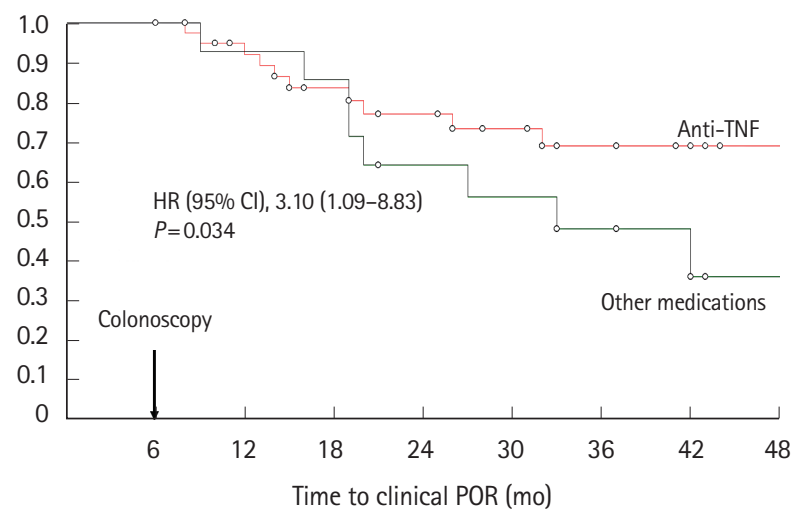

Fig. 5. Kaplan-Meier curve comparing the risk of clinical POR in patients treated with either anti-TNF or other medications while they did not receive any anti-TNF agent as prophylactic medication and experienced endoscopic POR at 6 months. HR and $P$-values are given in multivariable analyses with $95 \% \mathrm{Cl}$. HR, hazard ratio; $\mathrm{Cl}$, confidence interval; TNF, tumor necrosis factor; POR, postoperative recurrence.

prevent endoscopic POR and experienced endoscopic POR at 6 months, 27 were on thiopurines (azathioprine $[\mathrm{n}=20]$ and 6-mercaptopurine [ $\mathrm{n}=7]), 5$ were on methotrexate, 1 on tacrolimus, 5 on metronidazole and 17 had no medication. During a mean duration of follow-up of $28.9 \pm 16.1$ months, clinical POR occurred in $32.7 \%$ (18/55) of the patients. In multivariable analysis (Supplementary Table 4) adjusted on RI and use of immunosuppressive therapy as prophylactic medication, antiTNF was the best option to prevent clinical POR (HR, 3.10; 95\% CI, 1.09-8.83; $P=0.034$ ) (Fig. 5).

\section{DISCUSSION}

In this real-world cohort from a tertiary referral inflammatory bowel disease center, we confirmed that anti-TNF agents are the most effective option to prevent endoscopic and clinical POR in CD. In addition, we observed that combination therapy with anti-TNF and immunomodulatory agents was more effective than anti-TNF monotherapy in patients exposed to anti-TNF prior to surgery. Finally, we identified primary nonresponse to anti-TNF before surgery and prophylactic monotherapy as factors associated with anti-TNF failure to prevent endoscopic POR in patients with CD.

In our cohort, we analyzed anti-TNF effectiveness to prevent endoscopic POR in distinguishing the patients according to their exposition to anti-TNF prior to intestinal resection as this dichotomy has not been reported so far in the postoperative period. However, it seems to us relevant, as anti-TNF ther- apy is more effective in naïve patients to treat the traditional flare of $\mathrm{CD}^{17}$ In addition, the PREVENT trial suggested that patients who had not preoperatively been treated with antiTNF could be predictive of better efficacy to prevent clinical POR, even though the primary endpoint statistical significance was not reached (OR, 0.556; 95\% CI, 0.301-1.026). It is further noteworthy that in the PREVENT trial, the secondary endpoint of endoscopic recurrence of $\mathrm{RI} \geq \mathrm{i} 2$ was less in the infliximab-treated patients (30.6\% with infliximab vs. $60.0 \%$ with placebo; $95 \% \mathrm{CI}, 18.6 \%-40.2 \% ; P<0.001)$.

In anti-TNF-naïve patients, we report a very low rate of endoscopic POR (8.7\%) without difference in patients with or without concomitant immunosuppressive therapy (1/12 vs. 1/11). In multivariable analysis adjusted for the usual risk factors, we showed that anti-TNF was much more effective than immunosuppressive agents (thiopurines or methotrexate) (OR, 8.8; 95\% CI, 1.8-43.9; $P=0.008$ ) and no medication/ 5-ASA (OR, 5.2; 95\% CI, 1.0-27.9; $P=0.05$ ). These data are in line with those compiled in a meta-analysis from very smallsample size cohorts reporting endoscopic POR rate from $0 \%$ to $26.6 \%$ which was lower than controls including placebo, 5-ASA and thiopurines. ${ }^{9}$ It has been confirmed by 2 more recent network meta-analysis. ${ }^{18,19}$ In a non-randomized subanalysis of the POCER trial comparing 28 patients treated with adalimumab compared to 73 patients receiving thiopurines, the rate of endoscopic POR at 6 months was lower in patients treated with adalimumab (including $61 \%$ of anti-TNFnaïve patients and $100 \%$ of monotherapy) ( $21 \%$ vs. $45 \%, P=$ 0.028). In the PREVENT trial, only $17 \%$ of the 147 patients received concomitant thiopurines with infliximab and $25.3 \%$ of the patients were already exposed to anti-TNF before surgery. The rate of endoscopic POR at 18 months was lower in the group treated with infliximab compared to placebo (22.4\% vs. $51.3 \%, P<0.001)$. In our cohort from a referral center, $63 \%$ (199/316) of the patients were exposed to any anti-TNF prior to bowel resection. This higher rate of anti-TNF exposition is probably closer to the real-life practice as most of the patients currently receive at least 1 biologic before choosing the surgical option. Among these 199 patients, we showed, for the first time, that combination therapy with anti-TNF and immunosuppressive agents was more effective than anti-TNF monotherapy (OR, 2.32; 95\% CI, 1.02-5.31; $P=0.046)$ or other conventional medications (no medication, 5-ASA or immunosuppressive monotherapy) $(\mathrm{OR}, 3.65 ; 95 \% \mathrm{CI}, 1.40-9.58 ; P=0.016)$ to prevent endoscopic POR.

The use of anti-TNF mono or combined therapy was matter 
of debate during the last decade in the management of CD. The short-term benefit of combination therapy with infliximab and azathioprine was clearly demonstrated in the SONIC trial, which has included only patients without use of both thiopurines and anti-TNF before participating to the study. ${ }^{20}$ This benefit is more controversial in patients treated with adalimumab. ${ }^{21-23}$ In contrast, the long-term positive impact has been demonstrated for the 2 anti-TNF agents in reducing the risk of immunogenicity and preventing the risk of secondary loss of response. ${ }^{23}$ The impact of combination therapy was not previously investigated in the postoperative situation. The use of combination therapy could be supported by the higher risk of immunogenicity in patients previously exposed to at least 1 anti-TNF. ${ }^{24}$ We cannot evaluate whether pharmacokinetics and immunogenicity were related to the superiority of combination therapy, as we did not perform systematically drug monitoring in our center. However, the relevance of using drug monitoring in the postoperative management remains questionable and poorly investigated. ${ }^{25,26}$ We also focused on the factors associated with anti-TNF effectiveness in patients previously exposed to anti-TNF. Whether the history of anti-TNF use and response before surgery could have an impact on anti-TNF efficacy to prevent endoscopic POR is a highly relevant question, which has not been addressed so far. We showed that primary failure to at least 1 anti-TNF was associated with therapeutic failure. It is consistent with the data reported by a meta-analysis showing that primary nonresponse was associated with decreased likelihood of efficacy with a second anti$\mathrm{TNF}^{27}$ In this situation, the use of other biologics could be discussed even though the preliminary data on vedolizumab are disappointing ${ }^{28}$ while there is no data on ustekinumab so far.

As the risk of clinical POR is very high in case of endoscopic POR at 6 months, ${ }^{5,6}$ the efficacy of therapeutic escalation in this situation is a key point. In patients who did not receive any anti-TNF as prophylactic medication, we reported that anti-TNF is the most effective option to prevent clinical POR (OR, 3.10; 95\% CI, 1.09-8.83; $P=0.034$ ). These data are consistent with the data from Yamamoto et al. ${ }^{29}$ comparing 10 patients treated with continuous mesalamine ( $3 \mathrm{~g} /$ day), 8 patients treated with azathioprine therapy $(50 \mathrm{mg} /$ day), and 8 patients treated with infliximab ( $5 \mathrm{mg} / \mathrm{kg}$, every 8 weeks). The authors reported that no patient in the infliximab group, 3 patients (38\%) in the azathioprine group, and 7 patients $(70 \%)$ in the mesalamine group developed clinical recurrence (Crohn's Disease Activity Index $\geq 150)$, respectively $(P=0.01)$. In patients who received anti-TNF as prophylactic medications, we found that optimizing the anti-TNF (shortening the interval or increasing the dose) and adding an immunosuppressive agent in the same time is the best option to prevent clinical POR (OR, 7.38; 95\% CI, 1.54-35.30; $P=0.012$ ). Owing to the high risk of symptomatic recurrence in this situation, our data suggest maximizing the chance of success with the most potent option. In our cohort, we cannot draw any conclusion on the swap to another class of biological therapy to treat endoscopic POR and prevent clinical POR as only a few patients received these medications as rescue.

Our study has several limitations including that it is a single referral center with potentially more medically resistant $\mathrm{CD}$, absence of a central review process for endoscopic scoring and relatively small-sample size for some subgroups. In addition, this is a retrospective analysis randomization to compare therapeutic options. However, all our comparisons were adjusted on usual risk factors and potential confounders. Strengths of this analysis is the large real-world cohort with standardized management and evaluation of endoscopic POR by experienced inflammatory bowel disease physicians. We also were able to identify specific some factors associated with anti-TNF effectiveness to prevent endoscopic and clinical POR.

In conclusion, in this retrospective study we identified that anti-TNF was the most effective medication to prevent endoscopic and clinical POR. We also identified that primary nonresponse to anti-TNF therapy before surgery was associated with anti-TNF failure to prevent endoscopic POR. In case of endoscopic POR despite anti-TNF therapy, optimizing the anti-TNF combined with addition of immunosuppressive drug seems to be the best option to prevent clinical POR. We recommend consideration of combination of anti-TNF and immunosuppressive therapy in patients with pre-operative exposure to anti-TNFs in order to prevent recurrence.

\section{ADDITIONAL INFORMATION}

\section{Funding Source}

The authors received no financial support for the research, authorship, and/or publication of this article.

\section{Conflict of Interest}

Buisson A declares lecture fees for Abbvie, Amgen, Biogen, Ferring, Janssen-Cilag, Mayoli-Spindler, MSD, Pfizer, Roche, Takeda and Tillotts, Vifor Pharma and consulting fees for Abbvie, Amgen, Biogen, Celltrion, Janssen-Cilag, Mylan, Pfizer, Roche, Takeda and Tillotts. Rubin DT declares consultant fees 
for Abbvie, Abgenomics, Allergan, Inc., Amgen, Celgene Corporation, Forward Pharma, Genentech/Roche, Janssen Pharmaceuticals, Merck \& Co., Inc., Miraca Life Sciences, Napo Pharmaceuticals, Pfizer, Salix Pharmaceuticals, Inc., Samsung Bioepis, Sandoz Pharmaceuticals, Shire, Takeda and grant support from Abbvie, Genentech/Roche, Janssen Pharmaceuticals, Prometheus Laboratories, Shire, Takeda and UCB Pharma. The other authors declare no conflict of interest related to this work.

Rubin DT is an editorial board member of the journal but was not involved in the peer reviewer selection, evaluation, or decision process of this article. No other potential conflicts of interest relevant to this article were reported.

\section{Data Availability Statement}

Not applicable.

\section{Author Contribution}

Conceptualization: Buisson A, Rubin DT. Data curation: Buisson A. Formal analysis: Buisson A, Pereira B, Rubin DT. Investigation: Buisson A, Rubin DT. Methodology: Buisson A, Rubin DT. Supervision: Rubin DT. Visualization: Rubin DT. Writing original draft: Buisson A. Writing - review \& editing: Buisson A, Cannon L, Umanskiy K, Hurst RD, Hyman NH, Sakuraba A, Pekow J, Dalal S, Cohen RD, Pereira B, Rubin DT. Approval of final manuscript: all authors.

\section{ORCID}

Buisson A

Cannon L

Umanskiy K

Hurst RD

Hyman NH

Sakuraba A

Pekow J

Dalal S

Cohen RD

Pereira B

Rubin DT

https://orcid.org/0000-0002-6347-409X https://orcid.org/0000-0001-7621-1398 https://orcid.org/0000-0002-0209-3998 https://orcid.org/0000-0003-3459-1737 https://orcid.org/0000-0002-1390-8144 https://orcid.org/0000-0003-2519-6129 https://orcid.org/0000-0002-4790-8994 https://orcid.org/0000-0003-1207-0500 https://orcid.org/0000-0002-6114-8936 https://orcid.org/0000-0001-7640-1087 https://orcid.org/0000-0001-5647-1723

\section{Supplementary Material}

Supplementary materials are available at the Intestinal Research website (https://www.irjournal.org).

\section{REFERENCES}

1. Pariente B, Mary JY, Danese S, et al. Development of the Lé- mann index to assess digestive tract damage in patients with Crohn's disease. Gastroenterology 2015;148:52-63.

2. Peyrin-Biroulet L, Cieza A, Sandborn WJ, et al. Development of the first disability index for inflammatory bowel disease based on the international classification of functioning, disability and health. Gut 2012;61:241-247.

3. Peyrin-Biroulet L, Loftus EV Jr, Colombel JF, Sandborn WJ. The natural history of adult Crohn's disease in populationbased cohorts. Am J Gastroenterol 2010;105:289-297.

4. Buisson A, Chevaux JB, Allen PB, Bommelaer G, Peyrin-Biroulet L. Review article: the natural history of postoperative Crohn's disease recurrence. Aliment Pharmacol Ther 2012;35:625-633.

5. Rutgeerts P, Geboes K, Vantrappen G, Beyls J, Kerremans R, Hiele M. Predictability of the postoperative course of Crohn's disease. Gastroenterology 1990;99:956-963.

6. Buisson A, Cannon L, Umanskiy K, et al. Natural history of Crohn's disease postoperative recurrence in a US Referral Center in the era of biologics and therapeutic intensification based on early endoscopic findings. Gastroenterology 2018; 154:S1-S128.

7. De Cruz P, Kamm MA, Hamilton AL, et al. Crohn's disease management after intestinal resection: a randomised trial. Lancet 2015;385:1406-1417.

8. Buisson A, Chevaux JB, Bommelaer G, Peyrin-Biroulet L. Diagnosis, prevention and treatment of postoperative Crohn's disease recurrence. Dig Liver Dis 2012;44:453-460.

9. Carla-Moreau A, Paul S, Roblin X, Genin C, Peyrin-Biroulet L. Prevention and treatment of postoperative Crohn's disease recurrence with anti-TNF therapy: a meta-analysis of controlled trials. Dig Liver Dis 2015;47:191-196.

10. Gionchetti P, Dignass A, Danese S, et al. 3rd European evidencebased consensus on the diagnosis and management of Crohn's disease 2016: part 2: surgical management and special situations. J Crohns Colitis 2017;11:135-149.

11. Regueiro M, Schraut W, Baidoo L, et al. Infliximab prevents Crohn's disease recurrence after ileal resection. Gastroenterology 2009;136:441-450.

12. Regueiro M, Feagan BG, Zou B, et al. Infliximab reduces endoscopic, but not clinical, recurrence of Crohn's disease after ileocolonic resection. Gastroenterology 2016;150:1568-1578.

13. De Cruz P, Kamm MA, Hamilton AL, et al. Efficacy of thiopurines and adalimumab in preventing Crohn's disease recurrence in high-risk patients: a POCER study analysis. Aliment Pharmacol Ther 2015;42:867-879.

14. Savarino E, Bodini G, Dulbecco P, et al. Adalimumab is more effective than azathioprine and mesalamine at preventing 
postoperative recurrence of Crohn's disease: a randomized controlled trial. Am J Gastroenterol 2013;108:1731-1742.

15. Boucher AL, Pereira B, Decousus S, et al. Endoscopy-based management decreases the risk of postoperative recurrences in Crohn's disease. World J Gastroenterol 2016;22:5068-5078.

16. De Cruz P, Bernardi MP, Kamm MA, et al. Postoperative recurrence of Crohn's disease: impact of endoscopic monitoring and treatment step-up. Colorectal Dis 2013;15:187-197.

17. Colombel JF, Sandborn WJ, Rutgeerts P, et al. Adalimumab for maintenance of clinical response and remission in patients with Crohn's disease: the CHARM trial. Gastroenterology 2007; 132:52-65.

18. Singh S, Garg SK, Pardi DS, Wang Z, Murad MH, Loftus EV Jr. Comparative efficacy of pharmacologic interventions in preventing relapse of Crohn's disease after surgery: a systematic review and network meta-analysis. Gastroenterology 2015; 148:64-76.

19. Burr NE, Hall B, Hamlin PJ, Selinger CP, Ford AC, O'Connor A. Systematic review and network meta-analysis of medical therapies to prevent recurrence of post-operative Crohn's disease. J Crohns Colitis 2019;13:693-701.

20. Colombel JF, Sandborn WJ, Reinisch W, et al. Infliximab, azathioprine, or combination therapy for Crohn's disease. N Engl J Med 2010;362:1383-1395.

21. Matsumoto T, Motoya S, Watanabe K, et al. Adalimumab monotherapy and a combination with azathioprine for Crohn's disease: a prospective, randomized trial. J Crohns Colitis 2016;10: 1259-1266.

22. Reenaers C, Louis E, Belaiche J, Seidel L, Keshav S, Travis S. Does co-treatment with immunosuppressors improve out- come in patients with Crohn's disease treated with adalimumab? Aliment Pharmacol Ther 2012;36:1040-1048.

23. Kennedy NA, Heap GA, Green HD, et al. Predictors of antiTNF treatment failure in anti-TNF-naive patients with active luminal Crohn's disease: a prospective, multicentre, cohort study. Lancet Gastroenterol Hepatol 2019;4:341-353.

24. Roblin X, Vérot C, Paul S, et al. Is the pharmacokinetic profile of a first anti-TNF predictive of the clinical outcome and pharmacokinetics of a second anti-TNF? Inflamm Bowel Dis 2018; 24:2078-2085.

25. Fay S, Ungar B, Paul S, et al. The association between drug levels and endoscopic recurrence in postoperative patients with Crohn's disease treated with tumor necrosis factor inhibitors. Inflamm Bowel Dis 2017;23:1924-1929.

26. Wright EK, Kamm MA, De Cruz P, et al. Anti-TNF therapeutic drug monitoring in postoperative Crohn's disease. J Crohns Colitis 2018;12:653-661.

27. Gisbert JP, Marín AC, McNicholl AG, Chaparro M. Systematic review with meta-analysis: the efficacy of a second anti-TNF in patients with inflammatory bowel disease whose previous anti-TNF treatment has failed. Aliment Pharmacol Ther 2015; 41:613-623.

28. Yamada A, Komaki Y, Patel N, et al. The use of vedolizumab in preventing postoperative recurrence of Crohn's disease. Inflamm Bowel Dis 2018;24:502-509.

29. Yamamoto T, Umegae S, Matsumoto K. Impact of infliximab therapy after early endoscopic recurrence following ileocolonic resection of Crohn's disease: a prospective pilot study. Inflamm Bowel Dis 2009;15:1460-1466. 
See "Factors associated with anti-tumor necrosis factor effectiveness to prevent postoperative recurrence in Crohn's disease" on pages 303-312.

Supplementary Table 1. Risk Factors of Endoscopic Postoperative Recurrence in 117 Anti-TNF-Naïve Patients (Multivariable Analysis)

\begin{tabular}{lcc}
\hline Source & OR (95\% Cl) & $P$-value \\
\hline Active smoker & $1.168(0.339-4.027)$ & 0.806 \\
Perianal lesions & $0.561(0.173-1.823)$ & 0.337 \\
Prior bowel resection & $2.588(1.024-6.542)$ & 0.044 \\
Resection length $>30 \mathrm{~cm}$ & $1.524(0.636-3.650)$ & 0.345 \\
Surgery for refractoriness to medical therapy & $2.187(0.099-48.292)$ & 0.620 \\
Metronidazole to prevent endoscopic POR & $0.521(0.110-2.476)$ & 0.412 \\
Fistulizing phenotyp (B3) & $0.790(0.318-1.963)$ & 0.612 \\
Anti-TNF & Reference & 0.053 \\
5-ASA/no medication & $5.224(0.977-27.923)$ & 0.008 \\
Immunosuppressive therapy & $8.770(1.752-43.902)$ & \\
\hline
\end{tabular}

TNF, tumor necrosis factor; OR, odds ratio; $\mathrm{Cl}$, confidence interval; POR, postoperative recurrence; 5-ASA, 5-aminosalicylates. 
Supplementary Table 2. Risk Factors of Endoscopic Postoperative Recurrence in 199 Patients Exposed to Anti-TNF Prior to Surgery (Multivariable Analysis)

\begin{tabular}{lcc}
\hline Source & OR (95\% Cl) & $P$-value \\
\hline Female sex & $0.464(0.237-0.908)$ & 0.025 \\
Active smoker & $0.700(0.258-1.902)$ & 0.484 \\
Perianal lesions & $1.711(0.832-2.290)$ & 0.144 \\
\hline Prior bowel resection & $1.194(0.623-1.759)$ & 0.593 \\
Resection length $>30 \mathrm{~cm}$ & $1.636(0.842-3.178)$ & 0.147 \\
Surgery for refractoriness to medical therapy & $8.390(0.987-71.351)$ & 0.051 \\
Metronidazole to prevent endoscopic POR & $0.546(0.204-2.460)$ & 0.228 \\
\hline Fistulizing phenotype (B3) & $0.837(0.431-1.628)$ & 0.601 \\
At least 3 biologics before surgery & $2.676(1.012-7.079)$ & 0.047 \\
Combination therapy & Reference & 0.029 \\
Anti-TNF monotherapy & $2.450(1.099-5.482)$ & 0.032 \\
Conventional therapy & $2.631(1.078-6.381)$ & 0.333 \\
Anti-integrin therapy & $1.872(0.526-6.659)$ & 0.383 \\
Ustekinumab & $0.218(0.007-6.676)$ & \\
\hline
\end{tabular}

TNF, tumor necrosis factor; $\mathrm{OR}$, odds ratio; $\mathrm{Cl}$, confidence interval; $\mathrm{POR}$, postoperative recurrence. 
Supplementary Table 3. Multivariable Analysis of Predictors of Clinical POR in Patients Who Were Treated with Anti-TNF to Prevent Early Endoscopic POR in Crohn's Disease

\begin{tabular}{lcc}
\hline Factor & HR (95\% Cl) & $P$-value \\
\hline Female sex & $1.809(0.754-4.339)$ & 0.184 \\
Active smoker & $1.566(0.459-5.351)$ & 0.474 \\
Perianal lesions & $0.847(0.375-1.916)$ & 0.691 \\
Prior bowel resection & $1.260(0.539-2.945)$ & 0.593 \\
Exposure to anti-TNF before surgery & $0.925(0.179-4.783)$ & 0.926 \\
Primary nonresponder to 1 anti-TNF before surgery & $1.451(0.563-3.739)$ & 0.441 \\
Concomitant immunosuppressive drug & $0.737(0.330-1.644)$ & 0.456 \\
Fistulizing phenotyp (B3) & $0.520(0.220-1.228)$ & 0.136 \\
Optimizing anti-TNF+addition of immunosuppressive drug & $7.383(1.544-35.309)$ & 0.012 \\
Rutgeerts index $\geq i 3$ & $3.115(1.166-8.319)$ & 0.023 \\
\hline
\end{tabular}

$\mathrm{POR}$, postoperative recurrence; $T \mathrm{NF}$, tumor necrosis factor; $\mathrm{HR}$, hazard ratio; $\mathrm{Cl}$, confidence interval. 
Supplementary Table 4. Multivariable Analysis of Predictors of Clinical POR in Patients Who Were Not Treated with Biologics to Prevent Early Endoscopic POR in Crohn's Disease

\begin{tabular}{lcc}
\hline Factor & HR (95\% Cl) & $P$-value \\
\hline Concomitant immunosuppressive drug & $0.645(0.252-1.653)$ & 0.361 \\
Rutgeerts index & Reference & 0.264 \\
i2a & $2.508(0.500-12.585)$ & 0.191 \\
i2b & $3.166(0.563-17.820)$ & 0.047 \\
i3 & $9.387(1.026-85.916)$ & \\
i4 & Reference & 0.034 \\
Anti-TNF & $3.105(1.091-8.833)$ & \\
Other strategy & & \\
\hline
\end{tabular}

$\mathrm{POR}$, postoperative recurrence; $\mathrm{HR}$, hazard ratio; $\mathrm{Cl}$, confidence interval; $\mathrm{TNF}$, tumor necrosis factor. 